\title{
Excitons in Semiconducting superlattices, Quantum Wells, and Ternary Alloys
}

DE92 015178

\author{
M.D. Sturge \\ Professor of Physics \\ Dartmouth college \\ Hanover, NH 03755 \\ R.E. Nahory and M.C. Tamargo \\ Semiconductor Materials Research Group \\ Bellcore Inc. \\ Navesink Research and Enginee:-ing Center \\ Red Bank, NJ 07701-7020
}

This report covers the period sept 15, 1991 to May 31, 1992.

\section{contents}

Summary

Introduction

Work done under this grant in the budget period

1. Type II short-period AlAs/GaAs superlattices

(a) Use of spectroscopy in material quality control

(b) Temperature dependence of exciton dynamics

2. Band offsets in mixed type II-VI compound superlattices 6

3. Exciton-phonon coupling at isoelectronic traps in an indirect 6 gap semiconductor

Publications based on work supported by this grant

Personnel 


\title{
Excitons in Semiconducting Superlattices, Quantum Wells, and Ternary Alloys
}

\begin{abstract}
Summary
Semiconducting layered structures can now be fabricated with precisely defined layer thicknesses down to one monolayer. An example is the "superlattice" (SL) structure, in which two semiconductors with different band gaps are interleaved. The $($ tronic and optical properties of the SL are quite aifferent from those or the constituents and offer interesting new possibilities both in device design and in basic physics. This proposal aims to improve our understanding of optically excited states in SL's, particularly in the so-called "Type II indirect" SL's in which the electron and hole created by optical excitation are separated both in real and in momentum space. We study these structures by time-resolved tunable laser spectroscopy, with and without external perturvations such as magnetic field, electric field, and uniaxial stress.

In SIs with only a few atomic layers per period the familiar "effective mass model" ấ semiconductor states breaks down. We have made precise optical experiments on well-characterized material to test current "first principles" calculations of the band structure. Our work under this grant has shown that the material we are using is of sufficiently high quality to test the theoretical prodictions. Comparison of theory and experiment provides a new and sensitive probe of the interface quality on a fine scale. Statistical analysis of the temperature dependence of the exciton decay dynamics provides complementary information.

From a careful study of the exciton spectra of the recently discovered mixed type I- type II CdTe/CdznTe sLs we have obtained the band offset at the CdTe/CdZnTe interface to un recedented accuracy.

A new analysis of exciton-phonon coupling at isoelectronic traps, which provide a three-dimensional analog of the exciton traps formed by well width fluctuations in SIs, has corrected errors in the literatuie and given a self consistent model of phonon-assisted transitions at these centers.
\end{abstract}

\section{DISCLAIMER}

This report was prepared as an account of work sponsored by an agency of the United States Government. Neither the United States Government nor any agency thereof, nor any of their employees, makes any warranty, express or implied, $c$ : issumes any legal liability or responsibility for the accuracy, completeness, or usefulness of any information, apparatus, product, or process disclosed, or represents that its use would not infringe privately owned rights. Reference herein to any specific commercial product, process, or service by trade name, trademark, manufacturer, or otherwise does not necessarily constitute or imply its endorsement, recommendation, or favoring by the United States Government or any agency thereof. The views and opinions of authors expressed herein do not necessarily state or reflect those of the United States Government or any agency thereof. 


\section{Introtuation}

The new crystal-growth techniques of molecular beam epitaxy (MBE), metal-organic chemical vapor deposition (MOCVD) and related techniques, permit the fabrication of structures made up of atomicaliy flat layers of semiconductors such as GaAs and AlAs ${ }^{1}$. The electronic properties of such structures are quite different in many respects from those of the parent bulk materials. Study of these structures can give new insight into basic problems of solid-state physics, particularly those associated with low dimensionality, and have led to unexpected and fundamental discoveries, such as the integral and fractional quantum Hall effect. Furthermore, these structures are of great technological interest, since they give the device designer new options in, and unprecedented control over, the properties of the working material.

of the many physical techniques for the study of such structures, optical spectroscopy is unique in that it gives information about excited states as well as the ground state of the system ${ }^{2}$. Such information is obviously useful to the designer of opto-electronic devices; it is also essential to the understanding of the basic physics of these structures. The energies, wave functions and dynamics of electronically excited states are more sensitive than is the ground state to the physical assumptions of any model used to describe the system. Furthermore, through such phenomena as the Raman effect and phonon-assisted transitions, the atomic vibrations of the structure can be probed; these too differ greatly from those of the parent materials.

The fundamental optical process in an semiconductor or insulator is the creation or destruction of an electron-hole pair with the corresponding absorption or emission of a photon. The electron and hole attract each other and form a neutral bound entity called the exciton. At low temperature and low excitation intensity, transitions involving the creatic.. and destruction of excitons dominate the optical properties of reasonably pure crystals ${ }^{3}$. In a perfect system excitons move freely; in a real system the exciton dynamics give valuable insight into deviations from perfection (such as impurities, defects, interface roughness, and composition fluctuations) as well as into the exciton-phonon interaction.

In bulk semiconductors of technological interest, operating at room temperature or above, the small binding energy of the exciton permits the device designer to ignore excitonic effects, and the chief practical use of the optical spectroscopy of excitons has been as an analytic tool. In the layered structures that we will consider here, on the other hand, excitonic effects dominate the optical spectra even at room temperature, and are responsible for the non-linear optical response which is essential to the new generation of optical devices, such as logic gates and optical switches based on optical

bistability ${ }^{4}$. Thus the study of excitons in these structures has come to play an important technological role in the field of optoelectronics.

In this proposal we are primarily concerned with a particular structure, the "Type II superlattice" (SL). It consists of many thin (in our case, 3-30A) layers of GaAs, with a small bandgap, alternating 
with similar layers of AlAs, which has a larger bandgap 5,6 . AlAs is an indirect gap semiconductor; i.e. its conduction band (electron) minimum is not at the Brillouin zone center $\Gamma$, as is the valence band (hole) maximum, but is at or near the $x$ point on the zone boundary. If. the GaAs layer is less than about 11 monolayers thick (while the thickness of the AlAs layers is no less than that), the confinement energy of the electron is so large that the $\Gamma$ conduction band minimum $(C B M)$ in the GaAs is pushed up above the X CBM in the AlAs. Thus the lowest electron state is in the AlAs layer while the hole state remains in the GaAs layer. Hence the lowest exciton consists of the AlAs $X$ electron and the GaAs $\Gamma$ hole, and is indirect in momentum space as well as being spatially separated in real space, so that its decay by photoluminescence (PL) is forbidden to zero'th order by momentum conservation. The dynamics of exciton decay are found to depend critically on the deviation of the interface from perfect flatness.

We have paid particular attention to sis with periods $(m+n)$ of 8 monolayers or less. Until recently it was believed that such SLs would be indistinguishable, except perhaps for a slight anisotropy, from the bulk alloy, but this has been shown to be mistaken: as we shall see below, we have found that AlAs/GaAs SLs containing even only a single monolayer of each constituent, if of good quality, have an electron band structure fundamentally different from that of the bulk alloy. In these SLs the usual methods of calculating SI band structure based on the effective mass approximation, such as the envelope function method ${ }^{7}$ and the Kronig-penney model ${ }^{8}$, break down, and have been replacei with more or less success by a plethora of so-called "first principles" calculations ${ }^{9}$. We have found that a careful comparison of theory and experiment can cast a great deal of light on the quality, in particular the interface flatness, of the material under study. 


\begin{abstract}
Work dene under this grant in the budget peried
1. Type II short-period AlAs/GaAs superlattices

As detailed in the previous report, our work under this grant las established the symmetry and energy of the conduction band minimum $(C B M)$ in very short period SL's. In the period covered by this report we have collated and published most of our results (pubs $1,4,8,12)$. In collaboration with DrS L.N. Pfeiffer and R. Hull of A.T. \& $T$. Bell Labs, we have begun to apply these results to the evaluation and quality control of MBE material. We have also developed a statistical model for the delocalization of excitons in an attempt to understand the temperature dependence of the time decay of luminescence in these indirect gap SL's.
\end{abstract}

\title{
(a) Use of spectroscopy in material guality control
}

For an ideal $1 / 1$ SL (i.e. a SL consisting of successive single monolayers of AlAs and GaAs) there is agreement between band structure calculations done by many different methods that the conduction band minimum (CBM) derives from the L point of bulk GaAs9, 10,11. On the other hand, our data show unambiguously that the CBM derives from the $x$ point (pub. \#4). Comparison with the known band structure of the disordered alloy Alo.5 $\mathrm{Ga}_{0.5} \mathrm{As}^{12}$, and detailed band structure calculations13,14, show that this is to be expected if the SL is disordered, an interchange of about $30 \%$ of the Ga and Al atoms being necessary to produce the observed re-ordering of the states. There is also evidence from electron diffraction of such mixing ${ }^{15}$. On the other hand, our data show that the selection rules for a perfect superlattice are well obeyed [pub. \#8]. This discrepancy can be resolved if the atomic interchanges are assumed not to be random, but are restricted to those which preserve the space group symmetry of the ideal superlattice. Total energy calculations:6 suggest that this type of interchanged SL is energetically favored over the perfect SL, although the energy differences appear at first sight to be too small to be important at the growth temperature of the SI. While much work remains to be done, it appears that spectroscopy, in combination with theory, can provide a useful tool for the evaluation of interface quality and cast light on the mechanisms by which interface roughness develops (pub \#12).

We have also studied some SL's grown at different temperatures, following up a suggestion 17 based on Raman scattering data that, contrary to the received wisdom, low temperature growth favors flat interfaces. Our photoluminescence results do not support this idea, though there are still some ambiguities to be resolved.

\section{(b) Temperature dependence of exciton dynamics}

It was shown many years ago that the non-exponential time dependence of pulse-excited luminescence of indirect excitons, in an alloy semiconductor (AlGaAs) at low temperature, can be explained quantitatively in terms of $X \rightarrow \Gamma$ scattering of electrons by a random distribution of scattering centers ${ }^{18}$. More recently, it was shown that an analogous model accounts for the corresponding data on Type II SL's 6,19,20. While the temperature dependence of the decay was 
qualitatively understood in terms of delocalization of excitons, which leads to averaging over different sites and ultimately to exponential decay, quantitative understanding was lacking. We have adapted the dynamical theory of the averaging of chaotic light $2 \hat{i}$ to this problem and obtained a quantitative fit to the data, irom which exciton hopping rates as a function of temperature can be obtained (pubs. $\# 13,14)$.

\section{Band offsets in mixed tyoe II-VI compound quantum weIls}

In collaboration with the group of Dr Merle d'Aubigne of the CNRS Laboratory in Grenoble, France, we have analyzed the spectra of compensated strain CdTe/Cd.912n.09Te quantum wells (pub. \#9). This system is of interest because the "average" valence band offset $V_{a}$ (defined as the band offset in the absence of shear strain) is extremely small. As a result, shear strain, which splits the light hole ( $\mathrm{LH}$ ) from the heavy hole (HH) band and has opposite signs in the CdTe and $\mathrm{Cd} .91 \mathrm{Zn} .09 \mathrm{Te}$ layers, dominates the offset so that the LH and $\mathrm{HH}$ reside in different layers ${ }^{22}$. By a careful analysis of the LH-HH separation in a range of SL's we have been able to extract an accurate value for $V_{a}$, which is found to be zero within experimental error. Comparison with various calculations ${ }^{23}$ shows that there is probably a close cancellation between the "chemical" offset (the offset in the absence of strain) and the hydrostatic strain contribution. These two contributions to the cffset are shown to be in principle not separable by spectroscopic measurements on a heterostructure.

3. Exciton-phonen coupling at iscelectronic traps in an indirect gap semiconductor

I'he exciton bound to an isoelectronic (i.e. electrically neutral) trap such as nitrogen in GaP has much in common with an indirect exciton localized by width fluctuations in a quantum well, since these are 11 so neutral. Since the bound exciton has an energy which for any iven trap is well defined rather than depending on random luctuations, it can provide a useful model for a localized exciton. $\therefore$ our last progress report we showed that a new theory of the phonon $s$ idebands $^{24}$ of these bound excitons is internally inconsistent (pub \#) and is based on incorrect data (pub \#6). We have now developed an a. ternative theory of these sidebands which deals with the "momentumc nserving" and "configuration-coordinate" types of phonon coupling in a consistent manner (pub. \#11). 


\section{Rublicatiens}

The following publications, which have appeared or been submitted in the current budget period, are based wholly or in part on work supported by this grant.

Papers published in or submitted to refereed journals

1. $\Gamma \rightarrow \Gamma$ emission from indirect gap very short period GaAs/AlAs superlattices. Weikun Ge, Janet L. Mackay, L. N.Pfeiffer and K. W. West, J. Lumin. 50,133 (1991).

2. Gain and strong signal saturation of photoexcited quantum well structures. Y. Danker, E. Einkman, A.Ron, E.Cohen and M.D.Sturge, Proc. SPIE 1283 (Quantum Well and superlattice Physics III) p.326 (1991).

3. Observation of quantum confinement by stirain gradients. K. Kash, B.P.van der Gaag, D.D.Mahoney, A.S.Godz, L.T.Florez, J.P. Harbison and M.D.Sturge, Phys. Rev. Lett. 67, 1326 (1991)*.

4. Conduction band minimum of (GaAs) 1-(AlAs) s superlattices: relationship to X minimum of AlAs. Weikun Ge, W.D.Schmidt, M.D.Sturge, L. N. Pfeiffer and K. W. West, Phys. Rev. B44, 3432 (1991).

5. Acceptor-like bound exciton in semiconductors. Yong Zhang, Phys. Rev. B45, 6809 (1992).

6. Comment on "Radiative and nonradiative recombination of bound excitons in GaP:N, I. Temperature behavior of zero-phonon line and phonon sidebands of bound excitons", M.D.Sturge and E. Cohen, Phys. Rev. B45, 11370(1992).

7. Comment on "Radiative and nonradiative recombination of bound excitons in GaP:N, I and IV". Weikun Ge, Yong Zhang, Donglin Mi, Jiansheng Zheng, Bingzhang Yan, and Boxi Wu, Phys. Rev. B, in press.

8. Space group symmetry and parity of the conduction band minimum in very short-period GaAs-AlAs superlattices, Weikun Ge, W.D.Schmict, M.D.sturge, L. N.Pfeiffer and K. W. West, Solid State Commun. 82, 951 (1992)

9. Exciton binding energies and the valence band offset in mixed Type I Type II strained layer superlattices, P. Peyla, Y. Merle d'Aubigné, A. Wasiela, R. Romestain, H. Mariette, N. Magnea, H. Tuffigo, and M.D.Sturge, Phys. Rev.B 46, July 15, 1992, in press.

10. A Photoexcited Three-Component Electron-Hole Plasma in Type II GaAs/AlAs Quantum Wells, Janet L. Mackay, M. D. Sturge, M.- H. Meynadier, M. C. Tamargo, and J. L. de Miguel, submitted to Phys. Rev.

11. On the phonon sidebands of nitrogen bound excitons in GaP. Yong Zhang, Weikun Ge, Donglin Mi, J.S. Zheng, B. Z. Yan and B. X. Wu, to be submitted to Phys. Rev..

* Our work on strain-induced quantum confinement is now funded by onR and is excluded from this progress report. However, some of the work described in pub. 3 was done before the ONR grant began in April 1991, and was supported in part by the DOE grant. 


\section{Cenference abstracts}

12 Electron states in short period GaAs/AlAs superlattices (invited talk). Weikun Ge, Bull Am. Phys. Soc. 37,377 (1992)

13 A statistical analysis of radiative decay of localized indirect gap excitons. J.F.Angell and M.D.Sturge, Bull Am. Phys. Soc. 37, 241 (1992)

14 Time-resolved photoluminescence study of exciton localization in shortperiod superlattices. W.Ge, J.F.Angell, W.D.Schmidt and M.D.Sturge, Bull Am. Phys. Scc. 37, 605 (1992).

\section{Thesis.}

15 A photo-excited three component electron-hole plasma in type II GaAs/AlAs quantum wells. Janet $L$. Mackay, Ph.D. Thesis, Dartmouth College, October 1991.

References to other papers will be found below.

\section{Beferences}

1. See W.T.Tsang (ed.)"Lightwave Communications Technology, Part A: Material Growth Technologies" (Vol. 22A of "Semiconductors and Semimetals", Academic Press, New York, 1.985).

2. See Special issues on "Optical properties of semiconducting quantum wells and superlattices", ed. M.D.Sturge and M-H Meynadier, J. Lumin. 44, 199-414 (1989); ibid, 46, 66-155 (1990).

3. See, for example, E.I.Rashba and M.D.Sturge (eds.) "Excitons" (North Holland Press, Amsterdam, 1982).

4. H.M.Gibbs, "Controlling light with light" (Academic Press, New York, $1985)$.

5. P.Dawson, B.A.Wj.lson, C.W.Tu and R.C.Miller, Appl. Phys. Lett. 48, 541 (1986); B.A. Wilson, IEEE J. Quant. Electron. 24, 1763 (1988).

6. E.Finkman, M.D.Sturge and M.C.Tamargo, Appl.Phys. Lett. 49,1299 (1986); E. Finkman, M.D.Sturge, M.-H. Meynadier, R.E. Nahory, M.C.Tamargo, D.M.Hwang and C.C.Chang, J. Lumin. 39, 57 (1987).

7. G.B.1stard "Wave mechanics applied to semiconductor heterostructures", (Editions de Physique, les Ulis, 1988).

8. R. de L. Kronig and W.G.Penney, Proc. Roy. Soc. Iondon A130, 499 (1931).

9. For a review see L. J. Sham and Yan-Ten Lu, J.Lumin. 44, 207 (1989).

10. S-H Wei and A. Zunger, J. Appl. Phys. 63, 5794 (1988) 
11. S.B.Zhang, M.S.Hybertson, M.L.Cohen, S.G.Louie and D.Tomanek, Phys. Rev. Lett. B63, 1495 (1989); G.P..Srivastava, R. J. Gordon, and Z. Ikonic, Superlatt. Microstr. 9,43 (1991).

12. H.C.Casey and M.B.Panish "Heterostructure lasers", Part B, (Academic Press, $1978)$ p. 17.

13 A. Zunger and D. B. Laks, Phys. Rev. B45, 11411 (1992)

14. L.J.Sham and Y. T. Lu, unpublished.

15. R.Hull, private communication (1992)

16. D. B. Laks and A. Zunger, Bull. APS 37, 659 (1992), and private communication (1992).

17. J. Menendez, private communication to L.N.Pfeiffer (1992).

18. M.V.Klein, M.D.sturge and E. Cohen, Phys. Rev B25, 4331 (1982); M.D.Sturge, E. Cohen and R.A.Logan, Phys. Rev. B27, 2362 (1983).

19. F.Minami, K. Hirata, K.Era, T.Yao and Y. Masumoto, Phys. Rev. B35, $2875(1987)$.

20. M.D.Sturge, Janet L. Mackay, Colette Maloney and J.K.Pribram. J. Appl. Phys. 66, 5639 (1989)

21. M. Jakeman and E.R.Pike, J. Phys. AI, 128 (1968).

22. Y. Merle d'Aubigne, H.Mariette, H.Tuffigo, R.T.Cox, G.Lentz, Le Si Dang, J.I.Pautrat and A. Waliesa, J. Cryst. Growth 101, 650 (1990).

23. J. Tersoff, Phys. Rev. Lett. 56, 2755 (1986); C.G.van de Walle, Phys. Rev. 39, 1871 (1989).

24. X.zhang, K Dou, Q Hong and M Balkanski, Phys. Rev. B41, 1376 (1990);Q.Hong, X.Zhang, and K Dou, Phys. Rev. B41, 2931 (1990); : see also H.Chang (X.Zhang), C. Hirlimann, M. Kanehisa and M. Balkanski, Scientia Sinica (ser.A) 25, 942 (1982). 


\section{Rersonnel}

\section{Principal Investigators}

Professor M.D.Sturge spends approximately $25 \%$ of his time throughout the year on this research. 25,8 of his equivalent salary for the coming budget year, at the current rate and excluding fringe benefits, will be $\$ 20400$. The cost of this is borne by the grantee.

Dr R.E.Nahory is Manager and Dr Maria C. Tamargo is a member of the Semiconductor Materials Research Group of Bellcore. They not only provide much of the material used in this project but also provide an essential link to the related work at Bellcore.

Research associate professor

Dr Weikun Ge obtained his Ph.D from the University of Manchester, England, in 1983. He is on leave from the Institute of semiconductors in Beijing, where he is an Associate Professor, and is employed full time on this grant. His experimental work is on the energy levels of very short period superlattices; he is also doing theoretical work on the EL2 defect in GaAs, on exciton-phonon interactions at defects, and on hot carrier effects in quantum wells.

Graduate students

Janet L. Mackay, MS 1987 (Yale U.), was supported by this grant from July to October 1991, when she took her Ph.D. She is now empluyed by IBM, Endicott NY.

W.D.Schmidt, MS 1986 (Indiana U. of PA), was supported by this grant until september 1991. He is now employed full-time by A O Tech of southbridge $M A$ and is in process of writing his thesis.

James Angell, BS 1986 (E.Nazarene College), is supported by this grant. He will complete his thesis in August and will not be supported during the coming biddget year.

Yong Zhang, MS 1985 (Xiamen U., PRC). Originally an exchange student near completion of his Ph.D. in China, he transferred to Dartmouth in 1990 . He was then supported by this grant, but transferred in 1991 to ONR support.

Donglin Mi, BS 1987 (Beijing U.), MS 1989 (Xiamen U.). At present supported b: jartmouth College, he will transfer to DOE support in July.

Tim Gfroerer, MS 1991 (Georgia Tech). At present supported by Dartmouth college.

Yitong Gu, BS 1989 (Fudan U., PRC). At present supported by Dartmouth College. 

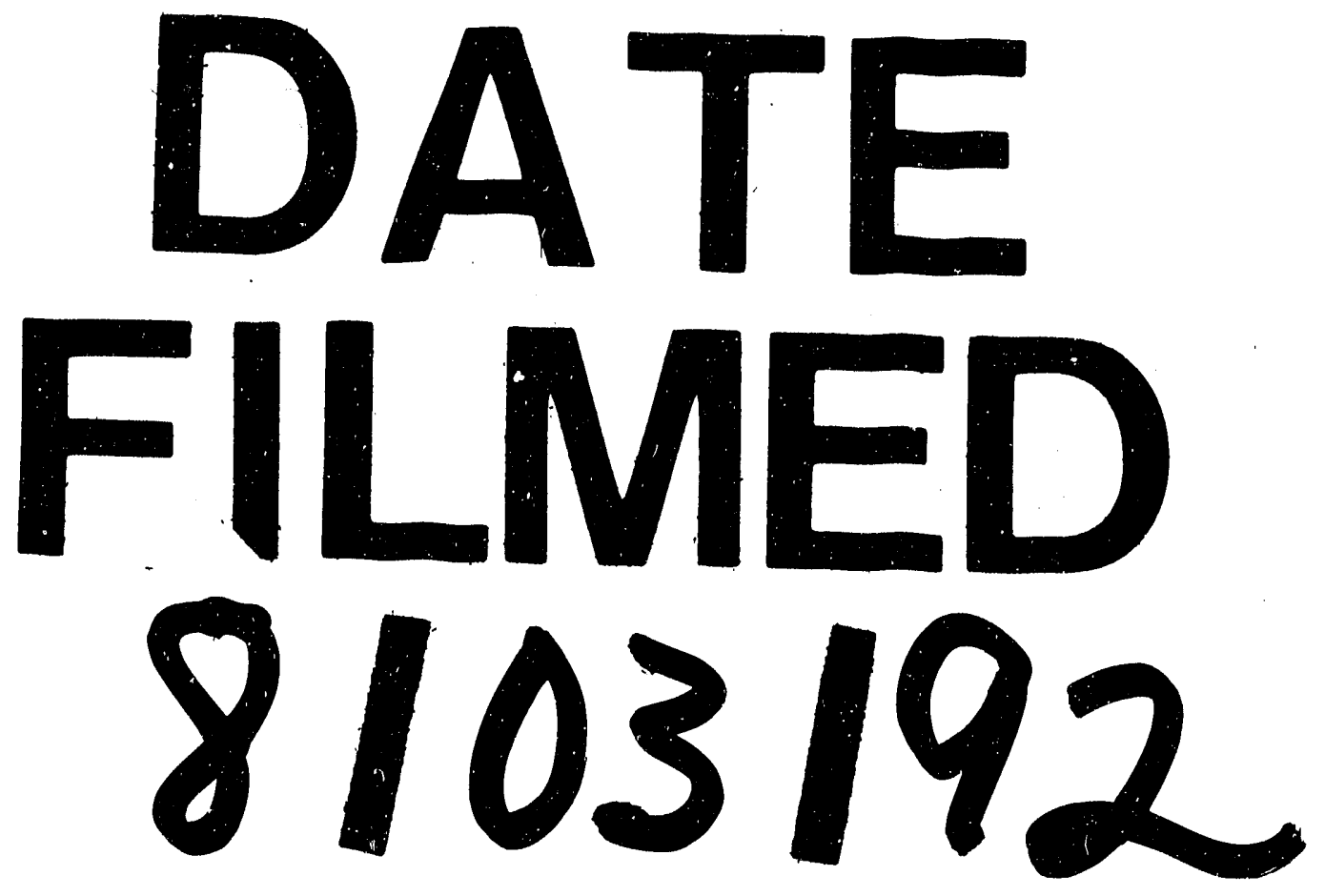
